\title{
Experimental analysis of cavitation in a centrifugal pump using acoustic emission, vibration measurements and flow visualization
}

\author{
Georgios Mousmoulis ${ }^{1,2, *}$, Nilla Karlsen-Davies ${ }^{2}$, George Aggidis², Ioannis \\ Anagnostopoulos ${ }^{1}$ and Dimitrios Papantonis ${ }^{1}$
}

${ }^{1}$ Laboratory of Hydraulic Turbomachines, School of Mechanical Engineering, National Technical University of Athens, Iroon Polytechniou 9, Zografou, Athens, 15780, Greece

${ }^{2}$ Lancaster University Renewable Energy Group, Department of Engineering, Lancaster University, Bailrigg, Lancaster, LA1 4YW, United Kingdom,

${ }^{*}$ Corresponding author. E-mail address: mousmoulisgeorge@ mail.ntua.gr

\begin{abstract}
The continuously increasing industrial productivity has resulted in a great breakthrough in the field of maintenance on centrifugal pumps in order to ensure their optimum operation under different operating conditions. One of the important mechanisms that affect the steady and dynamic operation of a pump is cavitation, which appears in the low static pressure zone formed at the impeller entrance region. This paper investigates the inception and development of cavitation in three different impellers of a laboratory centrifugal pump with a Plexiglas casing, using flow visualization, vibration and acoustic emission measurements. The aim of this study is the development of an experimental tool that detects cavitation in different impellers and the further understanding of the effects of blade geometry in cavitation development. The results show that the geometrical characteristics of the impeller affect cavitation development and behavior, while an acoustic emission sensor and an accelerometer can be applied for successfully detecting the onset of this mechanism.
\end{abstract}

Keywords: cavitation in centrifugal pumps; diagnostics and prognostics; flow visualization; noise and vibration measurements;

\section{Nomenclature}

\begin{tabular}{|c|c|c|c|}
\hline b & Blade width [mm] & $\mathrm{z}$ & Vertical distance between discharge and suction $[\mathrm{m}]$ \\
\hline$c_{n}$ & Mean radial flow velocity $[\mathrm{m} / \mathrm{s}]$ & $\beta$ & Blade angle [deg] \\
\hline $\mathrm{f}$ & Relative uncertainty [\%] & $\beta_{\mathrm{w}}$ & Relative flow direction [deg] \\
\hline $\mathrm{F}$ & Absolute uncertainty & $\lambda_{c}$ & Acceleration and friction losses coefficient [-] \\
\hline g & Acceleration due to gravity $\left[\mathrm{m} / \mathrm{s}^{2}\right]$ & $\lambda_{\mathrm{w}}$ & Geometrical and operating conditions coefficient [-] \\
\hline $\mathrm{H}_{\text {tot }}$ & Total head of the pump $[\mathrm{m}]$ & $\rho$ & Density $\left[\mathrm{kg} / \mathrm{m}^{3}\right]$ \\
\hline $\mathrm{H} v$ & Vapour Head [m] & $\sigma$ & Thoma number [-] \\
\hline $\mathrm{n}$ & Number of samples [-] & $\Phi$ & Flow coefficient [-] \\
\hline $\mathrm{n}_{\mathrm{q}}$ & Specific speed $[\mathrm{rpm}]$ & $\varphi$ & Incidence angle [deg] \\
\hline NPSH & Net positive suction head [m] & $\Psi$ & Head coefficient $[-]$ \\
\hline $\mathrm{P}_{\mathrm{st}}$ & Static pressure $[\mathrm{Pa}]$ & $\omega$ & Rotational speed [rpm] \\
\hline Q & Total flowrate of the pump $\left[\mathrm{m}^{3} / \mathrm{s}\right]$ & \multicolumn{2}{|c|}{ Subscripts } \\
\hline $\mathrm{Q}_{\mathrm{sp}}$ & Impeller flowrate $\left[\mathrm{m}^{3} / \mathrm{s}\right]$ & 1,2 & Leading/Trailing edge of the blade \\
\hline $\mathrm{Q}_{\mathrm{La}}$ & Leakage flowrate $\left[\mathrm{m}^{3} / \mathrm{s}\right]$ & Dis & Discharge pipe \\
\hline $\mathrm{R}$ & Radius [m] & $\mathrm{FC}$ & Fully developed cavitation operating point \\
\hline s & Standard deviation & $\mathrm{i}$ & Visual inception operating point \\
\hline $\mathrm{t}$ & Student parameter [-] & $\mathrm{r}$ & Random uncertainty \\
\hline $\mathrm{T}$ & Temperature $\left[{ }^{\circ} \mathrm{C}\right]$ & Suct & Suction pipe \\
\hline $\mathrm{u}$ & Circumferential velocity $[\mathrm{m} / \mathrm{s}]$ & s & Systematic uncertainty \\
\hline $\mathrm{v}$ & Mean flow velocity $[\mathrm{m} / \mathrm{s}]$ & $\mathrm{t}$ & Total uncertainty \\
\hline $\mathrm{W}$ & Relative flow velocity $[\mathrm{m} / \mathrm{s}]$ & $\mathrm{Y}$ & Measuring variable \\
\hline
\end{tabular}




\section{Introduction}

Cavitation is the hydrodynamic phenomenon of liquid vaporization due to local drop of static pressure, which is frequently created in the suction side of centrifugal pumps and mainly at the flow entrance into the impeller region, where the pressure becomes lower than the liquids vapour pressure. The development of vapour bubbles in such areas can be caused by incorrect pump installation (lower suction head), operation at higher than nominal rotation speeds or flow rates, increased transported fluid temperature, etc., and it is more likely to happen in conventional pumps without a special suction design. However, even without the above conditions, the high flow velocities developed in hydrodynamic machines could locally create favorable conditions for cavitation inception. After their formation, the vapour bubbles move along the flow path and reach higher pressure areas, where they are reliquified. The impulsive pressure waves during bubble implosion, as well as the water hammer pressures generated as the formed microjets impinge on the internal walls, significantly stress the material structure $[1,2]$. In addition, these pressure waves travel through the fluid and may excite structural vibration. After a certain operation time under such conditions the stressing can cause significant wear and material removal, which are followed by damage of hydraulic and mechanical components of the machine, such as the impeller blades, rotating shaft and bearings $[3,4]$. Furthermore, intense cavitation in the impeller changes the hydrodynamic shape of the blades creating flow recirculation and separation regions, which subsequently decrease the overall efficiency of the pump [5]. For all those reasons, scientists and engineers have developed various experimental methodologies in order to detect and study the onset and development of cavitation. These methods mainly include sound pressure and vibration measurements at a broad frequency range, and are sometimes followed by flow visualization. It should be noted that there are various noise and vibration sources in centrifugal pumps, which can be separated in two main categories; hydrodynamic and mechanical sources. Hydrodynamic sources relate to the flow characteristics such as the frequencies excited by turbulent flow and cavitation. Mechanical sources are linked to the rotating components of the system such as the rotational speed of the shaft (RF), the blade passing frequency (BPF), and the bearing frequencies.

Mc Nulty and Pearsall [6] used high frequency hydrophones and placed them at the suction and discharge sides of different pumps. The energy of a $40 \mathrm{kHz}$ signal obtained from the transducer at the inlet of the pump, showed a first peak in pressure well before the pump head starts to drop, which denoted the formation of the first bubbles close to impeller inlet. This phenomena was confirmed with the use of a stroboscope and a camera. In addition, it was observed that a further increase of vapour area decreased the noise level as a result of two mechanisms that acted on the pressure waves. On the one hand, the transition of the pressure waves from the liquid to the gaseous phase resulted in pressure transmission losses due to wave reflection [7] and at the same time the gaseous phase decreased the speed of sound due to compressibility reasons. Those factors contributed to the drop of the energy of the signal, when the two phase flow area enlarged. However, the total head of the pump was still unaffected at this point. Further reduction of the cavitation coefficient, caused a sharp reduction in head, while the noise continues to increase. Gopalakrishman [8] also used a high frequency Acoustic Emission (AE) sensor and presented noise energy at different frequency ranges as a function of Net Positive Suction Head (NPSH). Although the noise trend was the same for the different frequency ranges investigated and very similar to [6], the noise value appeared at a higher peak and cavitation detection was more apparent as the frequency band increased. Neil et al. [9] detected incipient cavitation with the use of AE sensors by measuring ultrasound energy of the signal at two locations. The position of the sensor relative to the cavitation area affected the noise emitted, although the onset of cavitation was depicted for both positions at the NPSH operating point when an increase in AE was observed. Alfayez et al. [10] measured AE activity with sensors 
placed in different positions in order to determine the best efficiency point (BEP) of the pump and detect cavitation. The sensor placed closer to the impeller eye identified cavitation inception and development as well as the BEP of the machine. According to their results, the development of large cavities resulted in noise reduction, however further decrease in the NPSH value increased the energy of the signal. The increase in noise at the minimum NPSH value was considered to be a result of the backflow phenomenon discussed thoroughly by Brennen [11]. The backflow phenomenon appears at low flowrates when the leakage flowrate between the tip of the blade and the casing becomes comparable with the impeller flowrate. Under such a condition, the cavities in the suction side are strengthened due to new bubbles that are formed in high speed leakage areas and recirculate in the inlet of the pump. For all the experimental works presented in $[6,8-10]$, the maximum high frequency noise level appeared far before the $3 \%$ total head drop.

Chudina [12] studied cavitation progress in different flowrates with the use of microphones in the audible frequency range. When the first bubbles appeared, the noise spectrum increased slightly both at high frequency ranges and at a few discrete frequencies. In parallel with Cernetic [13], he compared the monitoring of discrete half of BPF energy with the total noise level and showed that low frequency discrete tones are sufficient enough to detect the onset of cavitation regardless of the flowrate tested. Furthermore, Chudina et al. [14] used one microphone and tested three impellers with different geometrical characteristics. He showed that the frequency tone used for the monitoring of cavitation could be an arbitrary frequency in the audible frequency range. Similarly to [12], three different discrete tones were selected for each impeller and all of them appeared at a sharper increase than the total noise level curve. Cernetic et al. [15] presented the variation of the total head and relative amplitude of vibrations with the NPSH for two different pumps, one with a metallic closed impeller and one with a plastic semi-open impeller. The vibration results for the two impellers are for different frequencies; the metallic impeller is for a frequency band around $1600 \mathrm{~Hz}$ and the plastic for the discrete frequency of $148 \mathrm{~Hz}$. For both pumps, the vibration relative amplitude increased after the head drop but the vibration trend was different for each pump. In contrast to the semiopen impeller, where the vibration amplitude decreased and then rose again, the closed impeller pump did not appear to experience a noise reduction. Nevertheless, in [14] the noise measured for both impellers dropped sharply at the minimum NPSH tested. This indicates that different noise and vibration frequency bands, in the audible range, can detect cavitation inception. This was also validated in [16], where microphones and accelerometers appeared more practical in terms of installation and hence more appropriate in cavitation monitoring than high frequency hydrophones. In these studies, the noise and vibration appeared to have a slightly different behavior with change in material. The same was observed for the frequency band studied in relation to the geometrical characteristic of the impeller. For all the cases presented in [12-16], the maximum noise or vibration level coincided with a 3\% total head drop.

Zhang et al. [17] investigated vibration behavior with uniaxial and triaxial accelerometers in seven positions close to the pump under cavitation and non-cavitation conditions. During noncavitating conditions, the vibration energy was measured in a low frequency range and results showed that particularly at part load conditions the rotating stall increased the amplitude of the vibration energy. Under cavitating conditions, energy was measured in four different frequency ranges. Similarly to [8] the vibration energy trends were similar, however the higher frequency was more sensitive to the detection of the onset of cavitation. Summarizing all the previous work, significant progress has been made in the field of detection of cavitation with different techniques. The adequate selection of the frequency band of sound pressure and vibration measurements provided the information of inception of cavitation for a wide range of pumps. However, noise and vibration curves showed a slightly different trend as cavitation became more intense, mainly due to differences in impeller geometry and material, and the water 
quality. Finally different authors used flow visualization methods in order to study and detect cavitation [18, $19 \& 20]$. High speed video analysis was used for the study of cavitating flows in centrifugal pumps and authors managed to identify the onset and development of two phase flow $[18,20]$ as well as to measure the basic dimensions of the sheet cavity [19].

A novelty of the present work is the simultaneous application of noise and vibration measurements along with flow visualization on the same impellers, in order to compare directly the signals taken from the $\mathrm{AE}$ and accelerometer sensors with the photos obtained from the impeller, which, to our knowledge, has not reported in previous publications. This methodology and set up facilitates the investigation of the onset and development of two phase flow in the impeller, and makes possible to examine at which stage of the bubbles development, the sensors are able to identify their appearance. An additional contribution of this work is the testing of semi open impellers, whereas the majority of the experimental studies so far concern closed impellers. In this way, the present tests allow to study the backflow cavitation mechanism, together with the effect of the different geometrical characteristics of the blades on cavitation. The final aim of this ongoing research is the development of a reliable and accurate cavitation detection tool applicable to semi-open impellers of various geometric design and size. The three impellers examined here are made from the same material and are tested under similar cavitating conditions in the same test rig, whereas the casing of the pump is made from Plexiglas in order to visualize internal flow conditions.

\section{Experimental Set Up}

\subsection{Pump Lab Test Rig Description}

Experiments were carried out in a laboratory pump in the Engineering Department at Lancaster University [21]. The test circuit is sketched in Figure 1 and includes the main components of the pump with all the measuring devices. Water (blue line) is pumped from the tank and passes through the suction valve before it enters the centrifugal pump.

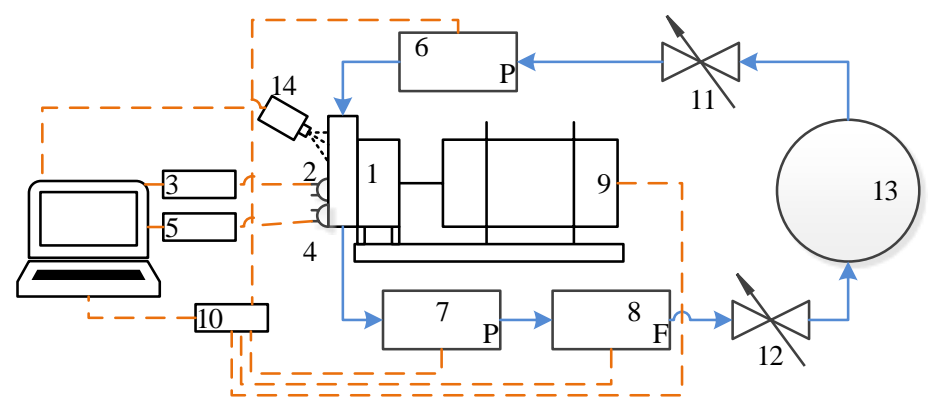

Figure 1 Sketch of the laboratory test set up configuration. 1. pump, 2. accelerometer sensor, 3. DAQ for vibration measurements, 4. AE sensor, 5. DAQ for AE measurements, 6. pressure transducer suction, 7. pressure transducer discharge, 8. flowrate sensor, 9. electric motor and rotational speed transducer, 10. DAQ for pressure, flowrate and rotational speed measurements, 11 . suction valve, 12. discharge valve, 13. water tank, 14. camera

The casing of the pump is made from Plexiglas and three semi open impellers were used in order to be able to visualize the flow. The $90^{\circ}$ bending pipe upstream of the impeller inlet disturbs the axial symmetry of the flow and creates an additional pressure drop. This results in a non-axisymmetric pressure and velocity field at the impeller inlet, and as a consequence cavitation inception is expected to appear at first on the left side of the impeller eye. A camera is fixed in position at the left side of the impeller in order to take comparable pictures for all examined cases. After the pump, the flow passes through the flowmeter and the discharge valve, which controls the flowrate, and returns to the main tank. Three different data acquisition (DAQ) systems were used as shown in Figure 1; one for rotational speed, flowrate and gauge pressure measurements, one for AE measurements, and one for vibration measurements. 


\subsection{Fluid Dynamic Definitions}

For the cavitation experiments the suction static pressure is progressively reduced in order to study the inception of the initial bubbles and their development. This is done by throttling the suction valve in order to increase the suction losses and reduce NPSH value, while properly regulating the discharge valve to retain the flow rate. After half minute from the opening of the discharge valve the flow is stabilized and the conditions are considered to be steady state. From this point and for $120 \mathrm{sec}$. the different set of data are acquired and after this, the new adjustment of the suction valve follows. During the various tests done for different $\sigma$ values, at this span of $120 \mathrm{sec}$., the macroscopic depiction of the cavitation area was remained almost unchanged. For that reason, we consider that the phenomenon appeared steady state characteristics in terms of its shape and the area that covers, even though microscopically exhibits transient features. The NPSH value is calculated for every operating point from equation (1):

$$
N P S H=H_{\text {tot_Suc }}-H_{V}=\frac{P_{\text {st_Suct }}}{\rho g}+\frac{v_{\text {suct }}^{2}}{2 g}-H_{V}
$$

where $\mathrm{H}_{\mathrm{V}}$ is the water vapor pressure as function of temperature, $\mathrm{P}$ is the absolute static pressure and $\mathrm{v}$ is the mean flow velocity in the suction pipe. For each impeller, four flowrates are tested and presented in results section as a percentage of flowrate $\Phi_{\mathrm{BEP}}$.

For each NPSH point, temperature, flow rate, gauge pressure, AE and vibration measurements are taken. Furthermore, for each operating point the flow field inside the impeller is captured in a photo with the use of a stroboscope and a DSLR camera. The rotational speed $\omega$, is kept constant at $1800 \mathrm{rpm}$ for all tests and the fluid temperature $\mathrm{T}$, at $23^{\circ} \mathrm{C}$. The tests are performed three times in order to ensure repeatability of measurements for the flow quantities, AE, vibration and the cavitation inception and development. Total relative uncertainty for all the measured and derived quantities is given in Table 1, while the evolution of $\mathrm{AE}$ and vibration, and the area of cavitation development inside the impeller were also repeatable. In this study the NPSH value is expressed in terms of the dimensionless Thoma's cavitation number:

$$
\sigma=N P S H / H_{t o t}
$$

where $\mathrm{H}_{\text {tot }}$ is the total head calculated from equation (3):

$$
H_{t o t}=H_{t o t_{-} D i s}-H_{t o t_{-} S u c}=\left(\frac{P_{s t_{-} D i s}-P_{s t_{-} S u c}}{\rho g}\right)+\left(\frac{v_{D i s}{ }^{2}-v_{S u c}{ }^{2}}{2 g}\right)+z
$$

where $\mathrm{z}$ is the vertical distance between the discharge and suction measuring points. In this study, the $\sigma$ and NPSH values are further specified with the use of a subscript that determines the intensity of cavitation. As a consequence, $\sigma_{\mathrm{i}}$ indicates the point where the first bubble becomes visible in the impeller flow path, and $\sigma_{\mathrm{FC}}$ the point where the impeller head is reduced drastically. Finally, in order to compare the three impeller geometries, the dimensionless parameters for the flowrate and head are introduced by equations (4) and (5), respectively:

$$
\Phi=c_{2 n} / u_{2}
$$

$$
\Psi=\left(2 g H_{t o t}\right) / u_{2}^{2}
$$

where $c_{2 n}$ is the mean radial flow velocity at the impeller outlet and $\mathrm{u}_{2}$ the circumferential velocity of the impeller.

The total relative uncertainty of each measured variable $\mathrm{Y}, \mathrm{f}_{\mathrm{t}, \mathrm{Y}}$ and its propagation in the derived quantities was calculated according to [22, 23]. As a consequence, $\mathrm{f}_{\mathrm{t}}$ of flowrate, suction and discharge static pressure, rotational speed and temperature are calculated through equation (6): 


$$
f_{t, Y}= \pm \sqrt{{f_{s, Y}{ }^{2}+f_{r, Y}^{2}}^{2}}= \pm \sqrt{f_{s, Y}^{2}+\left[\left(t \cdot s_{Y} / \sqrt{n}\right) / \bar{Y}\right]^{2}}
$$

where $f_{s}$ is the systematic uncertainty of the measuring device, $f_{r}$ is the random uncertainty associated with the mean value at the $95 \%$ confidence level, $\mathrm{s}_{\mathrm{Y}}$ is the standard deviation of the measured quantity $\mathrm{Y}, \mathrm{n}$ is the number of measurements, $\overline{\mathrm{Y}}$ is $\mathrm{Y}$ mean value and $\mathrm{t}$ is the Student parameter taken from [22]. Those errors are propagated in the calculations of $\Phi, \Psi$ and $\sigma$ and configure their levels of total uncertainty as it is shown in equations (7), (8) and (10) respectively:

$$
\begin{gathered}
f_{t, \Phi}= \pm \sqrt{f_{t, Q}^{2}+f_{t, \omega}^{2}} \\
f_{t, \Psi}= \pm \sqrt{f_{t, H_{t o t}}{ }^{2}+\left(2 \cdot f_{t, \omega}\right)^{2}}= \pm \sqrt{\left(F_{t, H_{t o t}} / \overline{H_{t o t}}\right)^{2}+\left(2 \cdot f_{t, \omega}\right)^{2}}
\end{gathered}
$$

where $\mathrm{F}_{\mathrm{t}, \mathrm{Htot}}$ is the total absolute uncertainty of total head calculated from equation (9):

$$
\begin{aligned}
& F_{t, H_{t o t}}= \pm \sqrt{\left(F_{t, P_{s t} \text { Suct }}\right)^{2}+\left(F_{t, P_{\text {st } \_ \text {Dis }}}\right)^{2}+\left(2 \cdot F_{t, Q}\right)^{2}} \\
& f_{t, \sigma}= \pm \sqrt{f_{t, N P S H}{ }^{2}+f_{t, H_{t o t}}{ }^{2}}= \pm \sqrt{\left(F_{t, N P S H} / \overline{N P S H}\right)^{2}+f_{t, H_{\text {tot }}}{ }^{2}}
\end{aligned}
$$

where $\mathrm{F}_{\mathrm{t}, \mathrm{NPSH}}$ is the total absolute uncertainty of NPSH calculated from equation (11)

$$
F_{t, N P S H}= \pm \sqrt{\left(F_{t, P_{\text {st_ Suct }}}\right)^{2}+\left(2 \cdot F_{t, Q}\right)^{2}+\left(F_{t, T}\right)^{2}}
$$

In this study the uncertainties related with the density $\rho$, gravity acceleration $\mathrm{g}$, the vertical distance $\mathrm{z}$ and the suction and discharge pipe diameters are considered insignificant and are ignored. Table 1 summarizes the total relative uncertainty for all the quantities measured and calculated through equations (6) to (11).

Table 1 Total relative uncertainty of all the measured and calculated variables

\begin{tabular}{|c|c|c|c|c|c|c|c|c|c|c|}
\hline & \multicolumn{5}{|c|}{ Measured Variables } & \multicolumn{5}{|c|}{ Calculated Variables } \\
\hline $\mathrm{Y}$ & $\mathrm{Q}$ & $\mathrm{P}_{\text {st_Suct }}$ & $\mathrm{P}_{\text {st_Dis }}$ & $\mathrm{T}$ & $\omega$ & $\Phi$ & $\Psi$ & $\sigma$ & $\mathrm{H}_{\text {tot }}$ & NPSH \\
\hline fy $[\%]$ & \pm 0.6 & \pm 0.3 & \pm 0.3 & \pm 1 & \pm 0.1 & \pm 0.6 & \pm 0.6 & \pm 0.8 & \pm 0.6 & \pm 0.6 \\
\hline
\end{tabular}

Finally, vibration value remained at acceptable levels and did not influenced the results presented in this study. More specifically, the velocity RMS at the range of 10 to $1000 \mathrm{~Hz}$ is lower than $3.2 \mathrm{mms}^{-1}$ for all the operating points, which according to [24] equals to newly commissioned general application rotordynamic pumps.

\subsection{Influence of Impeller Geometry on Cavitation Formation}

The performed tests include the investigation of the effect on cavitation of the blades in three different geometric designs, one with curved main and splitter blades (impeller 1), one with straight, radial blades (impeller 2), and one with curved, forward blades (impeller 3), all presented in Figure 2, while their main geometric and performance characteristics are tabulated in Table 2. Three key geometrical characteristics are investigated: the use of splitter blades, the inlet velocity triangle of the blade, and the effect of the blade width, $b_{1}$, which controls the tip clearance width (see Figure 3b). 


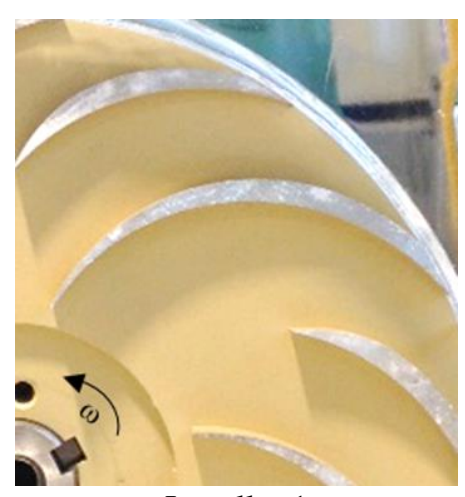

Impeller 1

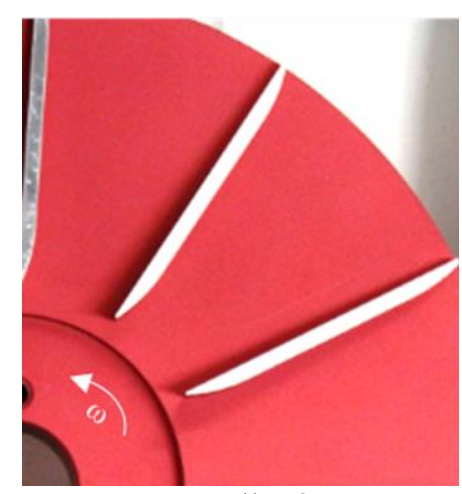

Impeller 2

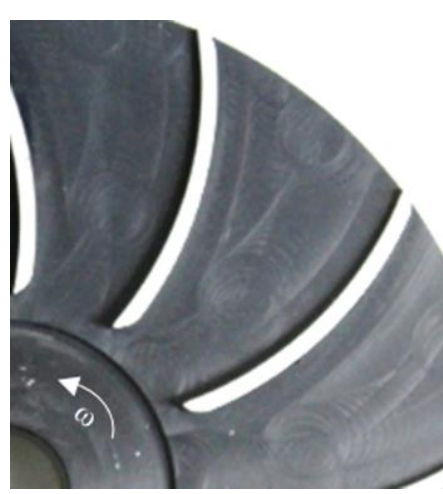

Impeller 3

Figure 2 The three impellers tested and their rotation direction

Table 2 Impellers Characteristics

\begin{tabular}{|c|c|c|c|c|c|c|c|c|c|c|}
\hline Impeller & $\begin{array}{c}\text { Specific } \\
\text { speed } \\
\mathrm{n}_{\mathrm{q}}[\mathrm{rpm}]\end{array}$ & $\begin{array}{l}\text { No of } \\
\text { Blades }\end{array}$ & $\begin{array}{c}\text { No of } \\
\text { Splitter } \\
\text { Blades } \\
\end{array}$ & $\begin{array}{c}\Phi_{\mathrm{BEP}} \\
{[-]}\end{array}$ & $\begin{array}{c}\Psi_{\mathrm{BEP}} \\
{[-]}\end{array}$ & $\begin{array}{c}\mathrm{b}_{1} \\
{[\mathrm{~mm}]}\end{array}$ & $\begin{array}{l}\text { Tip } \\
\text { clear. } \\
{[\mathrm{mm}]} \\
\end{array}$ & $\mathrm{R}_{1}[\mathrm{~m}]$ & $\mathrm{R}_{2}[\mathrm{~m}]$ & $\begin{array}{c}\omega \\
{[\mathrm{rpm}]}\end{array}$ \\
\hline No. 1 & 843 & 6 & 6 & 0.097 & 0.7 & 7.46 & 0.97 & \multirow{3}{*}{0.033} & \multirow{3}{*}{0.083} & \multirow{3}{*}{1800} \\
\hline No. 2 & 738 & 12 & 0 & 0.077 & 0.98 & 7.53 & 0.9 & & & \\
\hline No. 3 & 816 & 12 & 0 & 0.083 & 0.94 & 7.69 & 0.74 & & & \\
\hline
\end{tabular}

At this point, the equations that describe the cavitation development as a function of the velocity triangle of the inlet of the blade are given. Gulich [25] and Lobanoff [26] provide equation (12) in order to relate cavitation-free limit with the blade geometry and to estimate its value:

$$
N P S H=\left(\lambda_{c} c_{1 n}^{2}+\lambda_{w} w_{1}^{2}\right) / 2 g
$$

where, $\mathrm{c}_{1 \mathrm{n}}$ is the absolute and $\mathrm{w}_{1}$ the relative flow velocity at the blade inlet, as shown in the velocity triangle sketched in Figure $3 \mathrm{a}$. The coefficient $\lambda_{c}$ accounts for the acceleration and friction losses at the inlet, while $\lambda_{w}$ is depended on geometrical and operating parameters. The $\lambda_{c}$ can vary between 1.1 and 1.35 , whereas $\lambda_{\mathrm{w}}$ range from 0.1 to 2.5 depending on the cavitation criterion selected $\left(\sigma_{\mathrm{i}}, \sigma_{\mathrm{FC}}\right.$ etc. $)$ and the geometrical characteristics of the impeller [25].

The absolute inlet flow velocity $\mathrm{c}_{1 \mathrm{n}}$ is calculated from equation (13), where the total flow rate through the impeller, $\mathrm{Q}_{\mathrm{La}}$, is the sum of the pump flow rate $\mathrm{Q}$, the leakage flow rate $\mathrm{Q}_{\mathrm{sp}}$ and the balance flow $\mathrm{Q}_{\mathrm{E}}$ (Figure 3b). The latter is zero in the current study, since the impellers do not have any balance holes.

$$
c_{1 n}=\left(Q_{L a} / 2 \pi R_{1} b_{1}\right)=\left(Q+Q_{s p} / 2 \pi R_{1} b_{1}\right)
$$

where $R_{1}$ and $b_{1}$ is the impeller inlet radius and blade width, respectively. The relative velocity $\mathrm{w}_{1}$ is calculated from the inlet velocity triangle (Figure 3a), using the peripheral velocity of the impeller, $\mathrm{u}_{1}$, or the angular rotation speed, $\omega$ :

$$
w_{1}=\sqrt{c_{1 n}^{2}+u_{1}^{2}}=\sqrt{c_{1 n}^{2}+\left(\omega \cdot R_{1}\right)^{2}}
$$

A detailed view of the pump configuration at the impeller inlet is shown in Figure 3b, where the small tip clearance is also depicted. The same nozzle and bending pipe at the inlet was used for all three impellers, which have an equal inlet radius and as a result, a similar $\lambda_{c}$ value can be assumed [25] for the analysis. On the other hand, the $\lambda_{w}$ coefficient increases with an increase in incidence angle $\varphi_{1}$ [26], (shown in the sketch of Figure 3a), together with the flow relative velocity $\mathrm{w}_{1}$ at the BEP. 


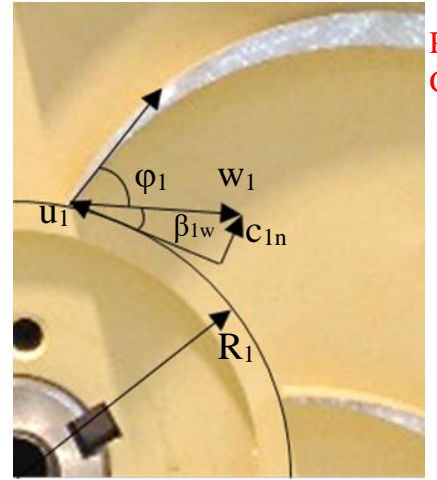

(a)

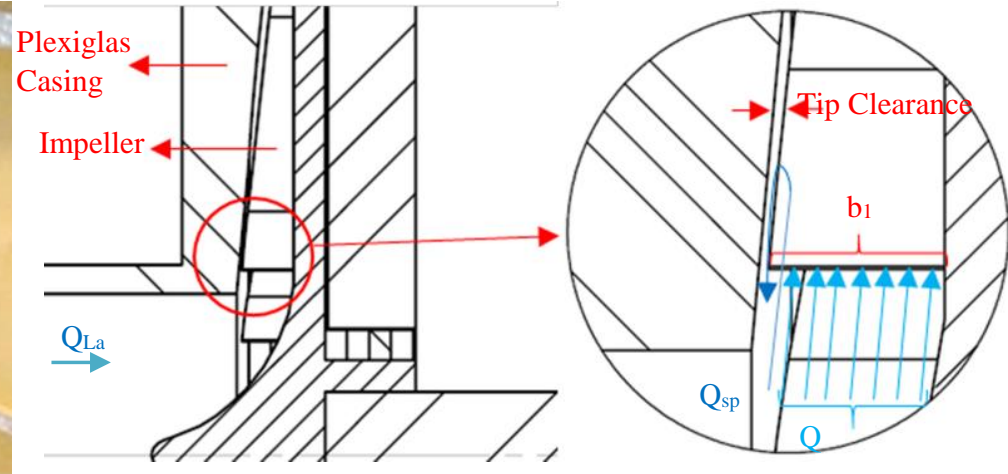

(b)

Figure 3 Velocity triangle at the inlet of impeller 1 (a), and detailed view of the blade inlet region and the tip clearance (b).

The incidence angle is calculated by equation (15), as the difference between the blade angle at the leading edge $\beta_{1}$ and the relative flow direction at the inlet, $\beta_{1 \mathrm{w}}$ (Figure $3 \mathrm{a}$ ):

$$
\varphi_{1}=\beta_{1}-\beta_{1 w}=\beta_{1}-\arctan \left(c_{1 n} / u_{1}\right)
$$

The values of $\varphi_{1}, c_{n 1}$ and $w_{1}$ as function of the flowrate are calculated and shown in Figure 4 for the three impellers. The incidence angle value $\varphi_{1}$ remains remarkably high, irrespective of the flowrate, because the inlet blade angle $\beta_{1}$ was designed to have high value in order to be easier to create and study the cavitation inception. Regarding the flow relative velocity, w1, Figure $4 \mathrm{~b}$ shows that it is much larger than $\mathrm{cn} 1$ for all the impellers, which highlights the greater effect of the second term of equation (12) in the prediction of critical NPSH, this is also discussed by Lobanoff [26].
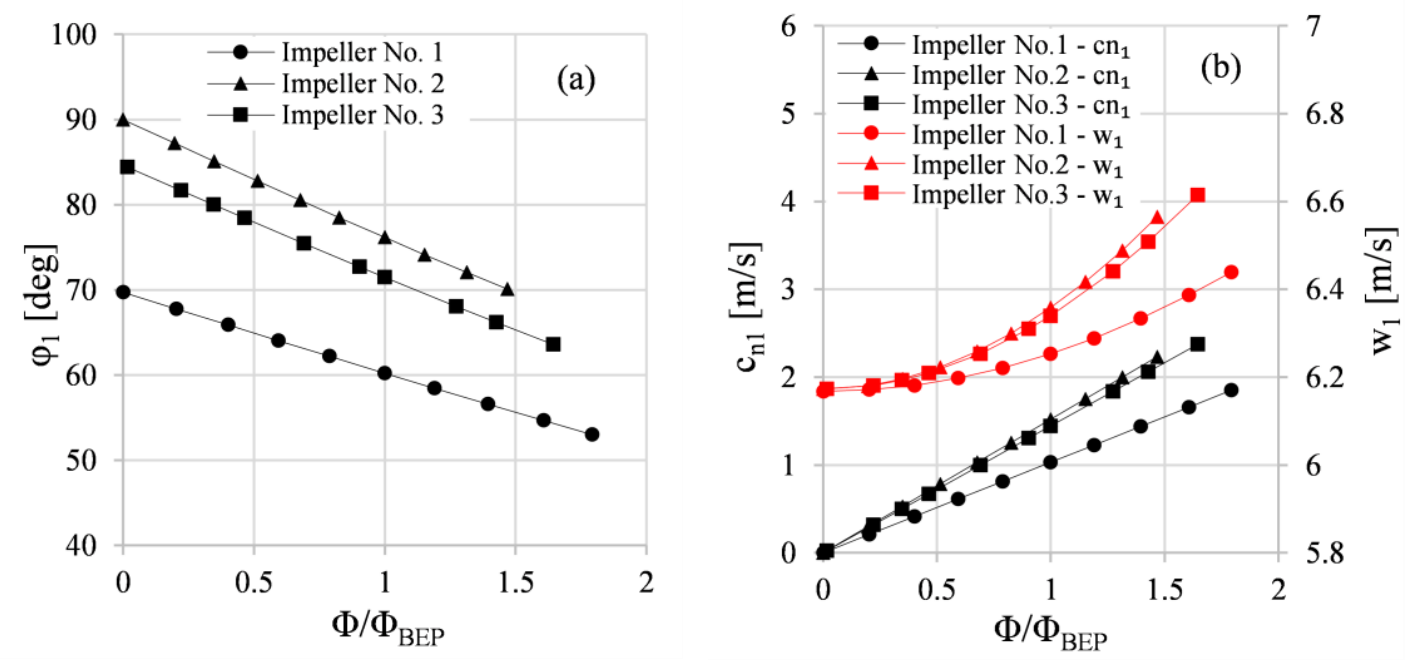

Figure 4 Variation range of a) incident angle $\varphi_{1}$, and b) flow velocities $\mathrm{c}_{\mathrm{n}}$, and $\mathrm{w}_{1}$ for the three impellers.

In addition, $\mathrm{w}_{1}$ values are similar for the three blades, especially for the partial flowrates that are tested, which designates the importance of incidence angle in any differences observed between the three geometries studied in this paper. Equation (12) is used as a qualitativetheoretical tool for the justification of the different visual, noise and vibration results between the different impellers tested.

\subsection{Acoustic Emission, Vibration Measurements and Flow Visualization}

Vibration and AE measurements were obtained in order to develop an experimental technique able to detect cavitation and study its development. The AE setup uses an integral preamp 
sensor and its signal is filtered in an analog band pass filter that allows the capture of frequencies in the range of $100-150 \mathrm{kHz}$ with a sampling rate, $\mathrm{N}$, of $306 \mathrm{kHz}$. After signal digitization, one second is divided in $\mathrm{p}=90$ segments, with $\mathrm{q}=3400$ values at each segment, and the root mean squared (RMS) is calculated. As a result, the average RMS of the segments at each second is calculated from equation (16):

$$
A E_{\text {RMS_ave }}=\left\{\sum_{i=1}^{p}\left(\sqrt{\frac{\left(V_{1}^{2}+\ldots+V_{q}^{2}\right)}{q}}\right)\right\} / p
$$

where $\mathrm{V}_{\mathrm{i}}$ is the $\mathrm{AE}$ raw data in Volt. Vibration measurements were carried out using a piezoelectric accelerometer able to measure frequencies up to $10 \mathrm{kHz}$. Its analog signal was filtered with the aid of an analog low pass filter with $10 \mathrm{kHz}$ cut-off frequency. Finally, the digitization of the vibration signal was done with a sampling frequency of $20 \mathrm{kHz}$ in order to satisfy the Nyquist theorem. Vibration signal processing includes the estimation of power spectral density (PSD) in $\left[\mathrm{m}^{2} /\left(\mathrm{s}^{4} \cdot \mathrm{Hz}\right)\right]$ units, based on the Welch estimate [27], and the calculation of the average power in different frequency ranges in $\left[\mathrm{m}^{2} / \mathrm{s}^{4}\right]$. For the PSD estimation, the time series of the signal is separated in five segments, and a Hanning window function is applied. The functions of Welch estimator for five segments and the band power are given in equations (17) and (18), respectively.

$$
\begin{gathered}
\hat{P}_{\text {Welch }}(\omega)=\frac{1}{5} \sum_{k=1}^{5} \frac{1}{L U}\left|\sum_{n=0}^{L-1} A[n] w[n] e^{-j \omega n}\right|^{2} \\
P_{\text {band limited }}=\frac{1}{\omega} \int_{\omega_{1}}^{\omega_{2}} \hat{P}_{\text {Welch }}(\omega) d \omega
\end{gathered}
$$

where $\mathrm{L}$ is the length of each segment, $\mathrm{A}[\mathrm{n}]$ is the digitized acceleration signal in time, $w[\mathrm{n}]$ is the window function and $\mathrm{U}$ is the window normalization constant.

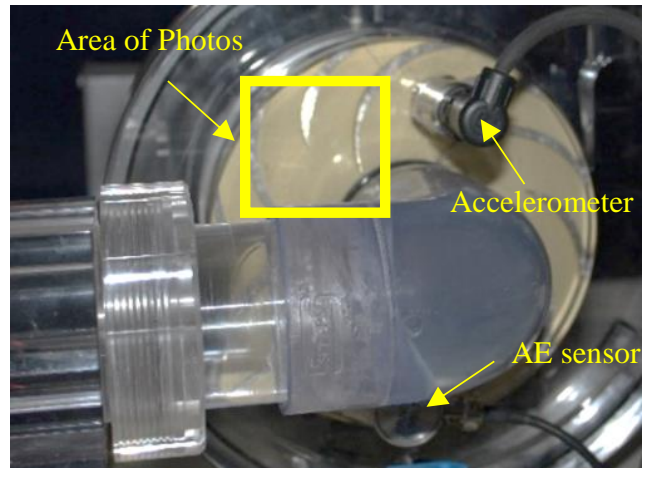

Figure 5 Positioning of $\mathrm{AE}$ and accelerometer sensors and of visualization window

The area of interest photographed with the camera as well as the positions of the AE and vibration sensors are depicted in Figure 5. Both sensors are located as close to the impeller eye as possible, as literature suggests [10]. The camera used in this setup is DSLR type with number of recorded pixels 4752 x 3168 , CMOS image sensor and max shutter speed of $0.25 \mathrm{msec}$. In the present study, flow visualization is used as a tool that confirms the existence of cavities, their onset as well as their extent and position. Flow visualization results are correlated with the level of the $\mathrm{AE}$ and vibration measurements, enabling the development of a cavitation inception criterion for all the impellers tested, associated with their geometrical characteristics. 


\section{Results and Discussion}

The results presented here include the variation of the total head $(\Psi)$, the vibration and $A E_{R M S}$ signals, and two phase flow pattern, as a function of the cavitation coefficient, $\sigma$. Firstly, the changes of total head for all flowrates tested are given and discussed. Afterwards, two flowrates are selected and photos of the cavitated flow in the impeller are included in order to demonstrate the intensity of the phenomenon in the different impeller geometries. The photos are followed by the noise and vibration results which provide useful information of the capabilities of the corresponding detection tools, as well as the effect of impeller geometry on cavitation onset and development.

\subsection{Total Head as function of $\sigma$}

Figure 6 shows the $\sigma-\Psi$ curves for the three impellers at four different flowrates, expressed with respect to the nominal flowrate $\Phi_{\text {BEP. }}$ In all cases the total head is not affected as long as $\sigma$ values remain above $0.3-0.35$, even after the appearance of the first bubbles at $\sigma_{i}$ which is depicted by the unfilled markers. The bubbles are observed visually. For further reduction of $\sigma$ down to the $\sigma_{\mathrm{FC}}$ value, the impeller head exhibits in all cases an abrupt and drastic drop. Irrespective of the impeller tested, as the flowrate increases both $\sigma_{\mathrm{FC}}$ and $\sigma_{\mathrm{i}}$ values become higher, due to the corresponding increase of flow velocities and the associated decrease of static pressures at the suction side of the pump.

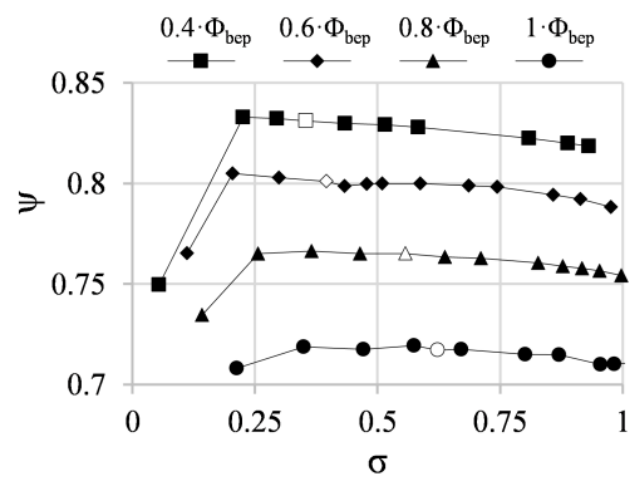

a) Impeller 1

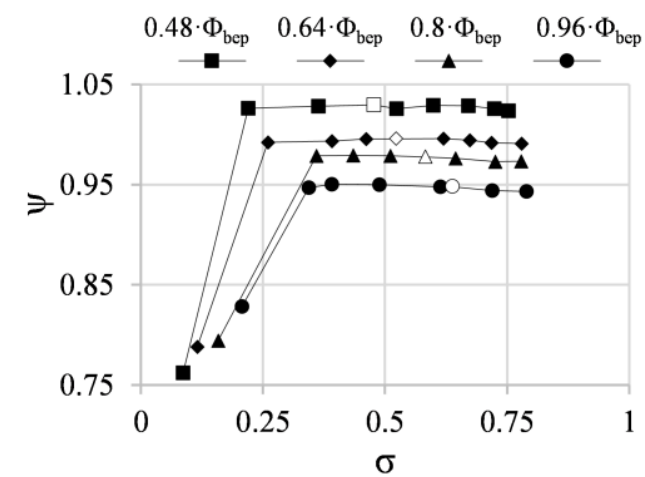

b) Impeller 2

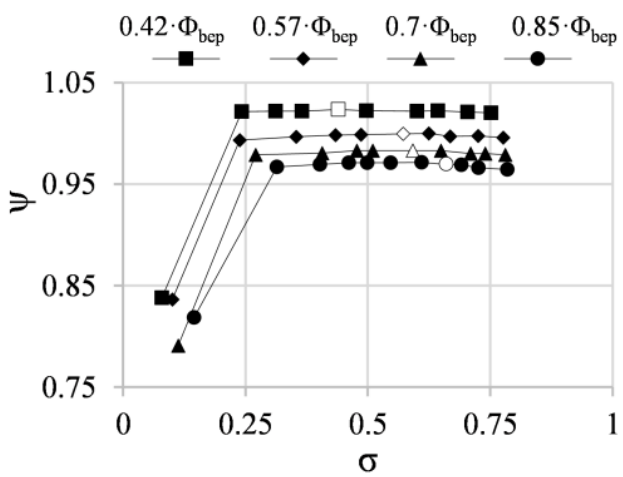

c) Impeller 3

Figure 6 Variation of $\Psi$, with the cavitation parameter, $\sigma$, of the three impellers, for various flowrates

For every measured point presented in Figure 6, $\mathrm{AE}_{\mathrm{RMS}}$, vibration measurements and photos from the flow path have been taken. This makes it possible to compare the behavior of the $\mathrm{AE}$ and accelerometer, as well as studying the onset and development of cavitation as a function of impeller characteristics and its influence on the corresponding signals. 


\subsection{Cavitation Visualization}

In Figure 7, two partial flowrates, approximately at 0.6 and $0.8 \cdot \Phi_{\mathrm{BEP}}$, are selected for every impeller in order to depict the onset and development of two phase flow. For every row (i.e. flowrate) in Figure 7 the photos of the internal flow field of four $\sigma$ values are presented. The $\sigma$ values include the $\sigma_{\mathrm{i}}$ and $\sigma_{\mathrm{FC}}$, as well as two other operating conditions between them $\left(\sigma_{\mathrm{m} 2}\right.$, $\left.\sigma_{\mathrm{m} 3}\right)$. In this way the development of cavitation from the inception point and first bubble creation, to the pump head drop point, where the two phase flow dominates is visualized. For all the cases tested, the first small bubbles always appear at the suction side of the leading edge (LE) of the blades, as it can be observed at $\sigma_{\mathrm{i}}$ operating point of Figure 7, the area that is more prone to develop minimum pressures that may allow local evaporation according to the theory [3, $5 \& 25]$. The two phase flow zone at the onset of the phenomenon is shown with the circles drawn on Figure 7.

For both partial flowrates presented, impeller 1 appears to have the lower $\sigma_{i}$ value. The reason for this behavior is the geometrical characteristics of the first impeller, which consists of six blades and six splitter blades, instead of twelve blades in the case of the second and third impeller. This results in a larger cross sectional area of flow passage at the inlet region of the first impeller, and subsequently lower flow velocities and higher static pressures. In addition, the first impeller has forward curved blades at the inlet, and hence the lowest flow incidence angle, $\varphi_{1}$ (see Figure 4), which, according to equation (12), improves the NPSH value and makes the pump less prone to cavitation.

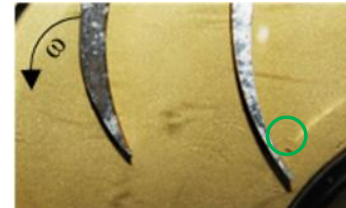

$\sigma_{\mathrm{i}}=0.4$

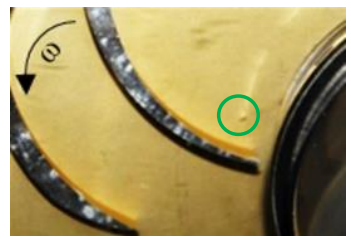

$\sigma_{\mathrm{i}}=0.55$

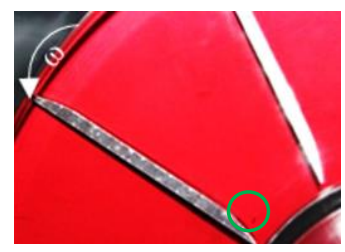

$\sigma_{\mathrm{i}}=0.52$

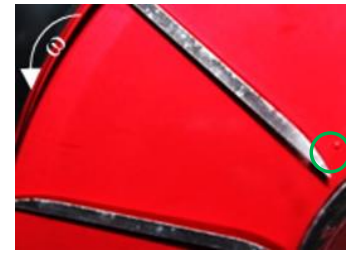

$\sigma_{\mathrm{i}}=0.58$

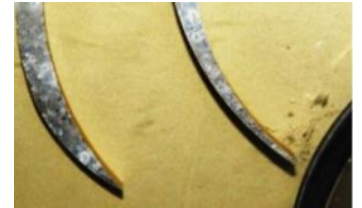

$\sigma_{\mathrm{m} 2}=0.3$
Impeller 1 - 0.6. $\Phi_{\mathrm{BEP}}$

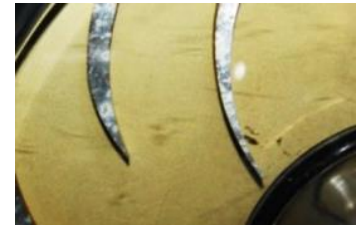

$\sigma_{\mathrm{m} 2}=0.46$

Impeller $1-0.8 \cdot \Phi_{\mathrm{BEP}}$

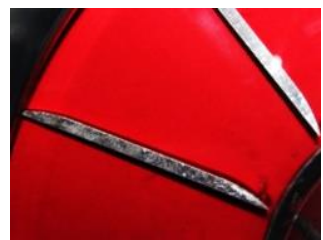

$\sigma_{\mathrm{m} 2}=0.39$

Impeller 2 - 0.64. $\Phi_{\mathrm{BEP}}$

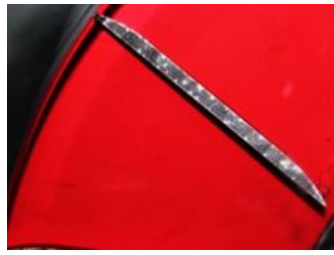

$\sigma_{\mathrm{m} 2}=0.43$

Impeller $2-0.8 \cdot \Phi_{\mathrm{BEP}}$

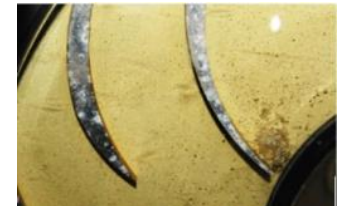

$\sigma_{\mathrm{m} 3}=0.2$

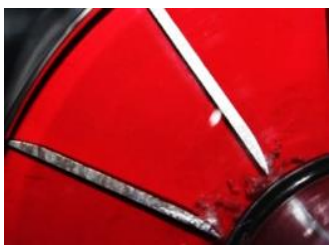

$\sigma_{\mathrm{m} 3}=0.26$

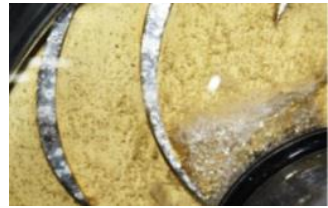

$\sigma_{\mathrm{FC}}=0.11$

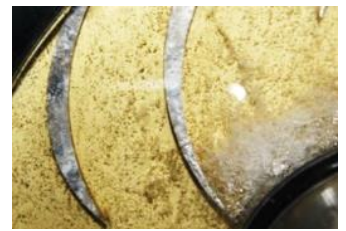

$\sigma_{\mathrm{FC}}=0.14$

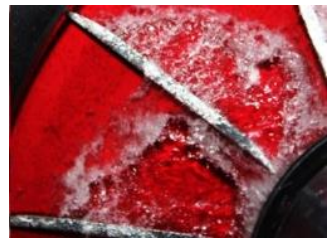

$\sigma_{\mathrm{FC}}=0.11$

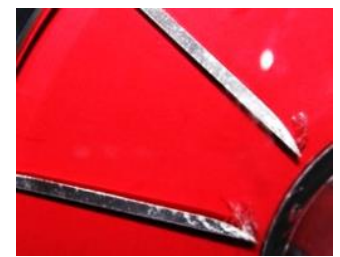

$\sigma_{\mathrm{m} 3}=0.36$

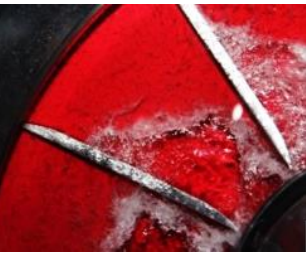

$\sigma_{\mathrm{FC}}=0.16$ 


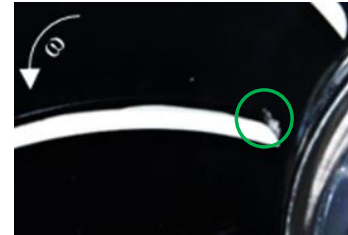

$\sigma_{\mathrm{i}}=0.57$

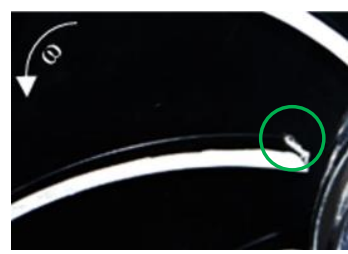

$\sigma_{\mathrm{i}}=0.66$

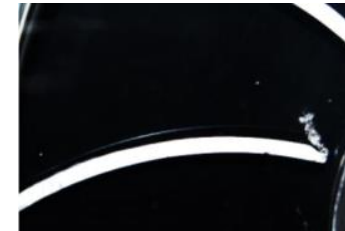

$\sigma_{\mathrm{m} 2}=0.43$

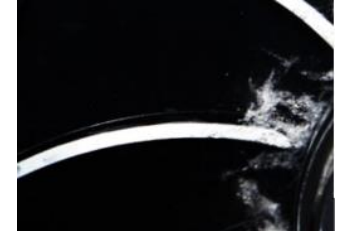

$\sigma_{\mathrm{m} 3}=0.24$

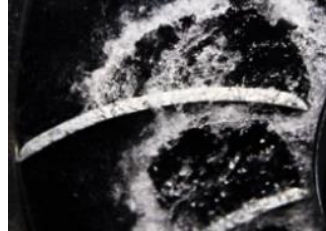

$\sigma_{\mathrm{FC}}=0.1$

Impeller $3-0.57 \cdot \Phi_{\mathrm{BEP}}$

Figure 7 Flow visualization for each impeller in four $\sigma$ numbers and two fractions of $\Phi_{\mathrm{BEP}}$

A reduction in the cavitation coefficient value increases the number of bubbles generated at the suction side of the blade, irrespective of the impeller design, as shown in the intermediate snapshots of Figure 7, for $\sigma_{\mathrm{m} 2}$ and $\sigma_{\mathrm{m} 3}$. In the end, further reduction of $\sigma$ value causes an abrupt and drastic head drop due to the extended size of cavitation area between the blades (Figure 7, $\left.\sigma_{\mathrm{FC}}\right)$. At this operating conditions, the flow becomes very unsteady and exhibits intensive recirculation and separation regions, a behaviour also observed by other researchers (e.g. Nelik [28]). Furthermore, at $\sigma_{F C}$ point, and depending on the flowrate, three cavitation sources may coexist: a) vapour cavities formed at higher $\sigma$ numbers inside the impeller due to static pressure drop at the suction side of the blade inlet and are already presented at Figure 7 ; b) vapour cavities generated at the suction valve and transferred to the elbow and at the inlet of the impeller; and c) backflow cavitation through the blade leakage area that typically appears at semi-open impellers [11]. Cavitation development at $\sigma_{\mathrm{FC}}$ is violent and extended, and almost blocks the suction of the impeller. In addition, the comparison of the flow inside the impellers at a similar flowrate and $\sigma_{\mathrm{FC}}$ values supports the previous discussion on the positive effect of splitter blades in impeller 1. At these operating points the two phase flow area of impeller 1 is less developed (mainly close to leading edge), compared to the other two impellers (unsteady area extends from leading to trailing edge).

\section{$3.3 \mathrm{AE}$ and vibration measurements}

\subsubsection{Vibration band power investigation}

The use of band power in the vibration measurements is used as the signal processing tool for the study of the onset and development of cavitation. However, before the analysis of vibration results, it is necessary to justify the choice of the frequency range selected for the calculation of band power. The vibration measuring system used in this study, which was described in detail in section 2.4, makes it possible to measure frequencies up to $10 \mathrm{kHz}$. In addition, the selection of the frequency range that will be used for the band power calculation is based on the study of the Welch estimator of the PSD [27], described in equation (17). The spectrum of the vibration signal in two flowrates for each impeller is presented in Figure 8. At each subplot three operating points are shown; the normal operation without cavitation (black color), the first bubble visual inception point (green color), and an operating point with cavitation (red color). For all the operating points, it is clear that fully developed cavitation excites most of the frequency spectrum, regardless of the impeller tested. The trend of the Welch estimator is similar with those presented in other works $[12,14 \& 15]$ at the same frequency range, with the use of microphones. At the range of $0-3 \mathrm{kHz}$ the amplitude differences are indiscernible due to the cavitation interaction with the low frequency mechanical noise caused mainly by the BPF 
and its harmonics [15]. However, at higher frequencies those harmonics become weaker and the broadband noise increases due to a more pronounced cavitation effect (Figure 8). The increase of amplitude starts at about $3 \mathrm{kHz}$ but becomes systematically sharper in the $5-10 \mathrm{kHz}$ range. As expected, the difference in amplitudes are less distinct between the cavitation onset and no cavitation operating points (green and black curves, respectively), but for the majority of the studied cases they are still recognizable. This indicates that the calculation of the band power frequency in the range of $5-10 \mathrm{kHz}$ can detect the inception of cavitation, as it was discussed also in [17]. Similar behaviour is observed for the remaining flowrates tested in this study, though their spectrum is not presented here.
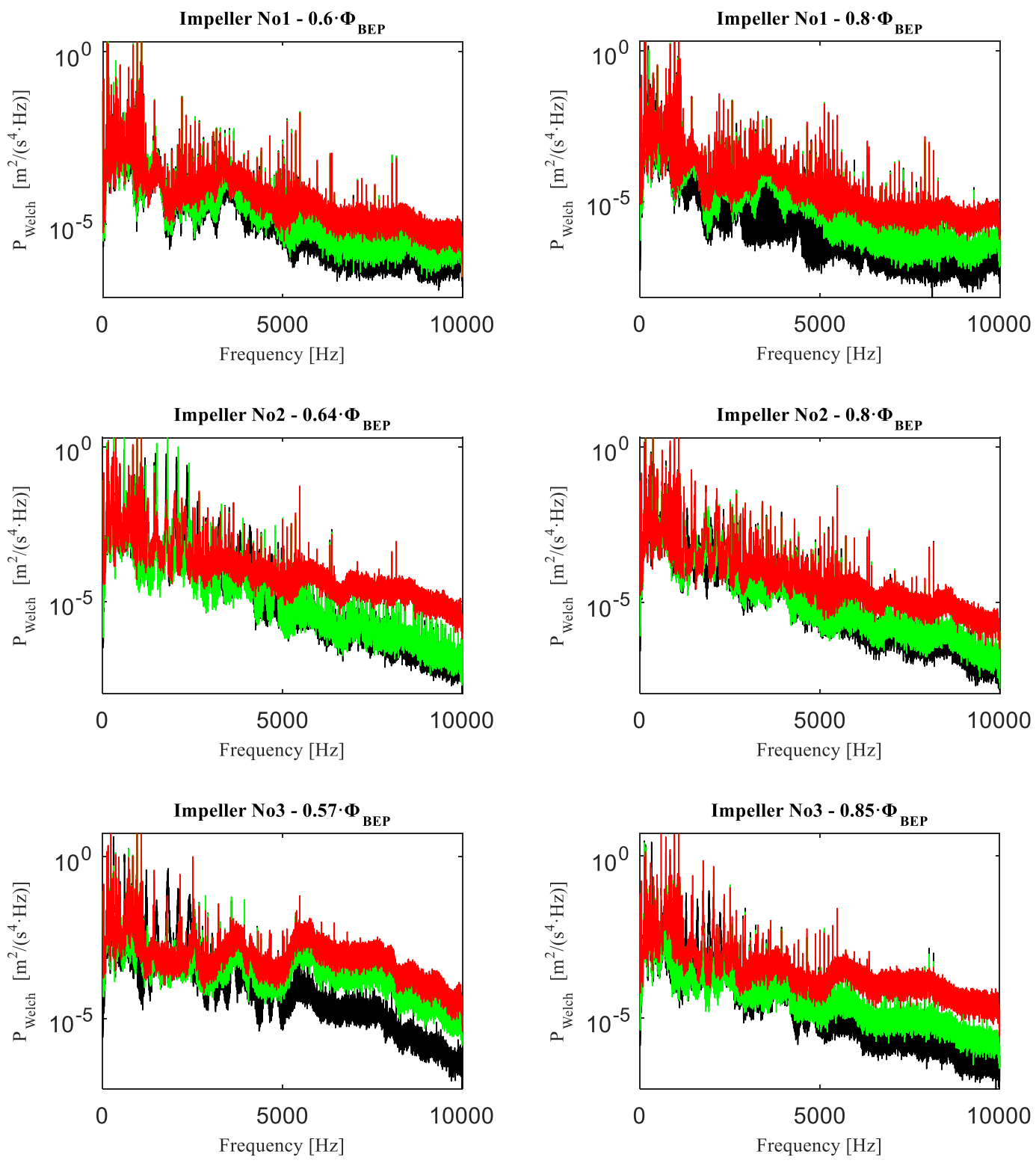

Figure 8 Spectrum of vibration measurements for two flow rates of each impeller, at three different $\sigma$ values: no cavitation $\left(\sigma>\sigma_{\mathrm{i}}\right.$, black), visual inception ( $\sigma \mathrm{i}$, green), and developed cavitation $\left(\sigma<\sigma_{\mathrm{i}}\right.$, red)

\subsubsection{AE and vibration results}

Figures 9, 10 and 11 show the $\mathrm{AE}_{\mathrm{RMS}}$ and vibration band power calculations as a function of $\sigma$, where the unfilled marker at each flowrate depicts again the $\sigma_{\mathrm{i}}$. Similarly to Figure 6 , the critical $\sigma$ value where both the vibration band power and the $\mathrm{AE}_{\mathrm{RMS}}$ raise, becomes higher as the flowrate increases. The visual inception point is consistent with the photos in Figure 7, and for 
all tests the $\mathrm{AE}_{\mathrm{RMS}}$ value starts to increase at the point of the first bubble appearance. The high frequency range energy captured by the $\mathrm{AE}$ sensor shows a remarkable sensitivity and capability to detect the first bubbles implosion, in accordance with Gulich's findings [25]. In addition, impeller 1 exhibits by far the lowest $\mathrm{AE}$ and vibration ranges for similar flowrates due to the effect of the splitter blades discussed in section 3.2.
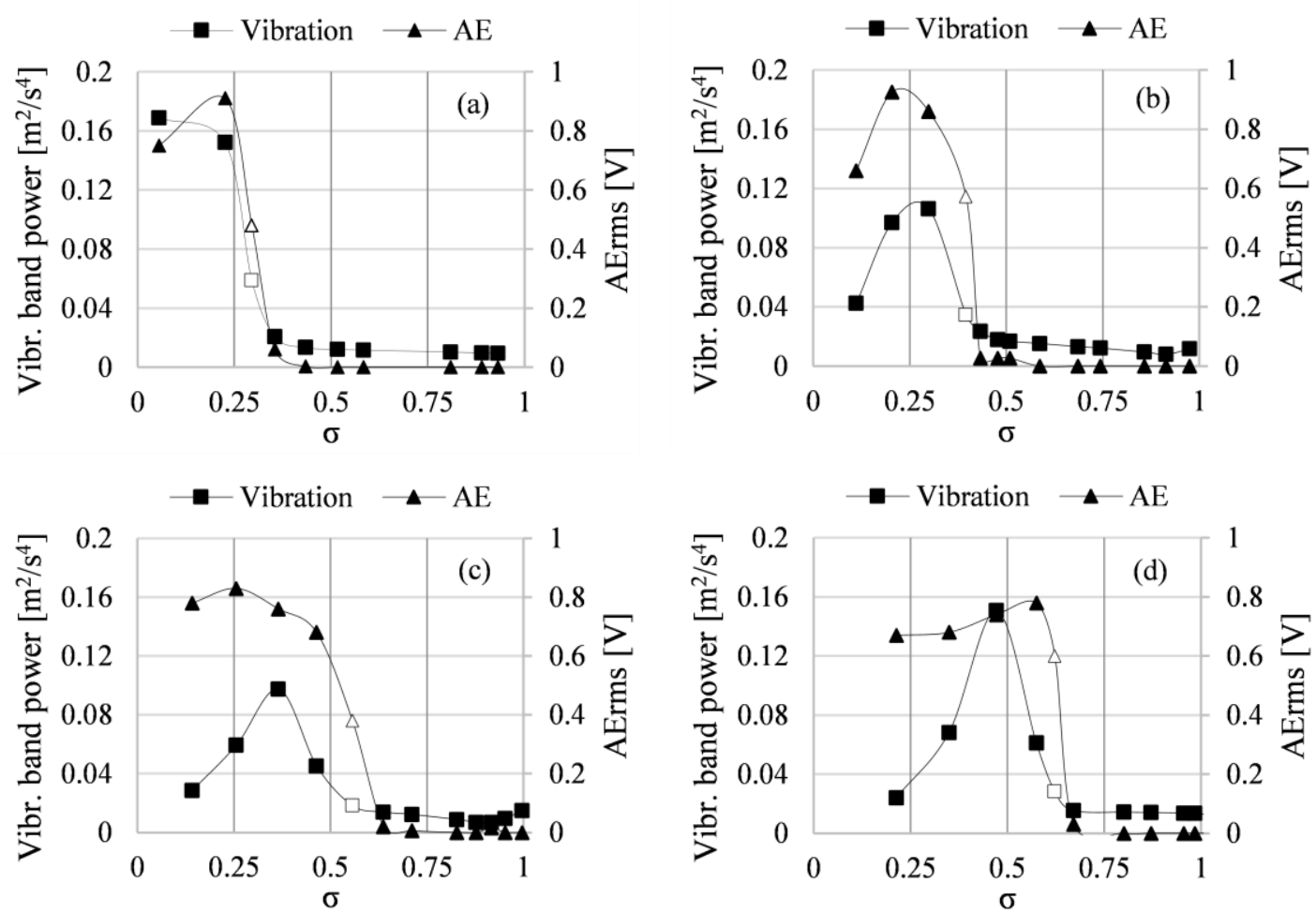

Figure 9 Noise and vibration as function of $\sigma$ for Impeller 1 and flowrate: a) $\left.\left.0.4 \cdot \Phi_{\mathrm{BEP}}, \mathrm{b}\right) 0.6 \cdot \Phi_{\mathrm{BEP}}, \mathrm{c}\right) 0.8 \cdot \Phi_{\mathrm{BEP}}$ and d) $1 \cdot \Phi_{\mathrm{BEP}}$

The incremental trend of $\mathrm{AE}_{\mathrm{RMS}}$ at the point of visual inception is sharper for the first and third impeller (Figures 9 and 11) than in the second (Figure 10). However, in the latter impeller the appearance of the first bubble was difficult to capture with the camera because the vapour area was extremely small and its appearance was not stable. Nevertheless, when $\sigma$ value decreases further, cavitation appearance is stabilized and the $\mathrm{AE}_{\mathrm{RMS}}$ values increase more sharply (Figure 10). Also, for 0.43 and $0.85 \cdot \Phi_{\mathrm{BEP}}$ at impeller 3 , a very slight increase in $\mathrm{AE}_{\mathrm{RMS}}$ was noticed for $\sigma$ values higher than $\sigma_{\mathrm{i}}$, for two possible reasons. Either bubbles do appear but the camera/stroboscope cannot capture them due to the very high frequency of creation/implosion or cavities are created in the elbow before the pump inlet, as discussed in [6] and [25].The vibration band power drawn in the same Figures 9 to 11, exhibits a very similar trend to the $\mathrm{AE}_{\mathrm{RMS}}$ values for the majority of the operating conditions tested. However, the increase of the band power starts at slightly lower $\sigma$ values than $\sigma_{\mathrm{i}}$, especially for the higher flow rates (Figures 9c, 10a, b, c, d and 11c, d).

This behavior is the result of the lower frequency range used for vibration analysis compare to $\mathrm{AE}$, which includes low frequency mechanical noise. Further reduction of $\sigma$ value results in increased number of bubbles, though the cavitation area remains small and restricted. This could be observed in Figure 7, where photos taken for $\sigma_{\mathrm{m} 2}$ values are presented. The enlargement of the two phase flow area is accompanied with an increase in noise and vibration signals, irrespective of the impeller and the flowrate (Figures 9 to 11). The vibration band power curve follows the trend of the AE curve and in almost all cases both curves reach a local maximum point. The local maximum point as a function of flowrate behaves differently for the 
$\mathrm{AE}$ and vibration measurements and for the various impellers, and hence, it is difficult to extract specific correlations.
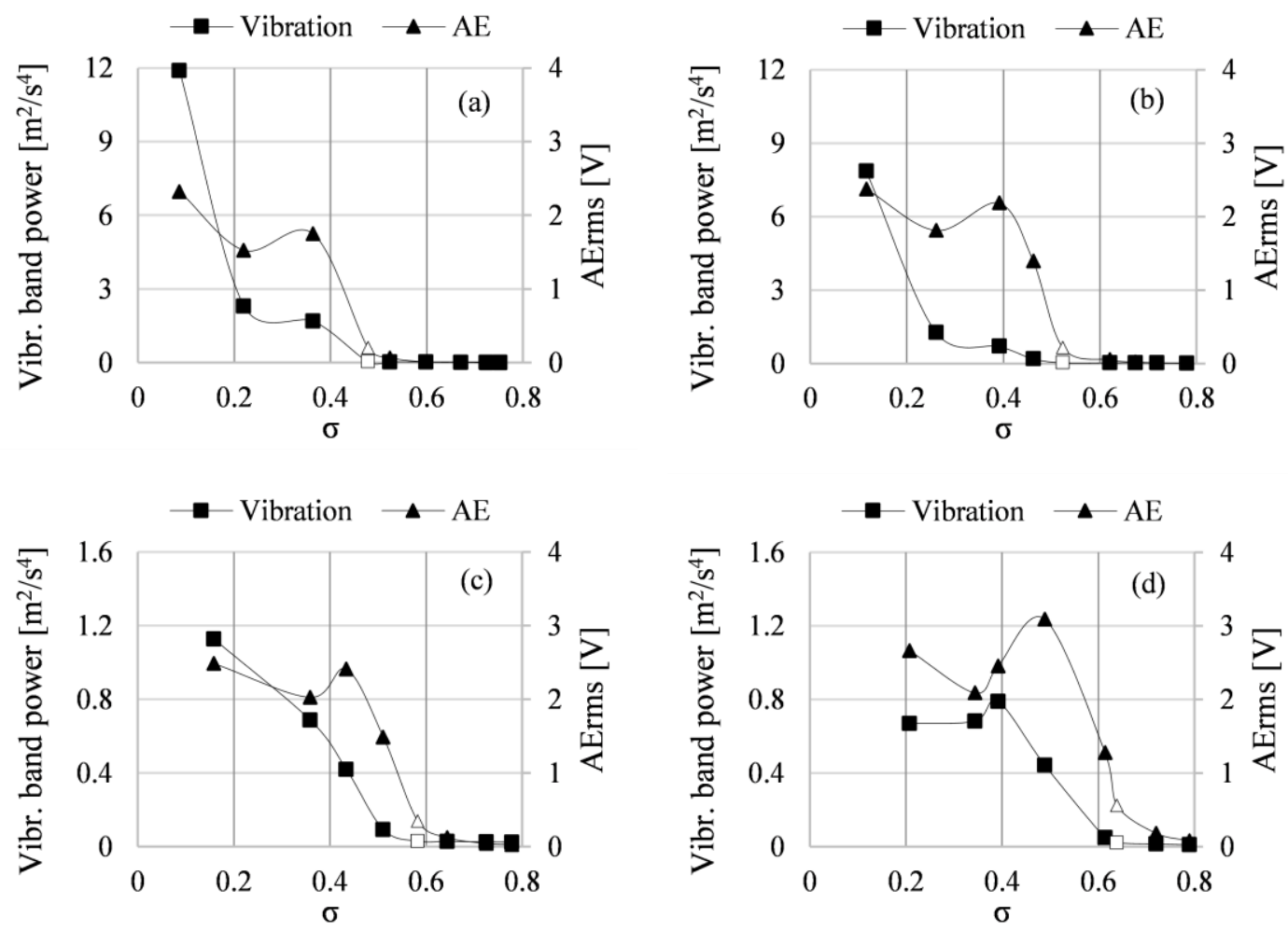

Figure 10 Noise and vibration as a function of $\sigma$ for Impeller 2 and flowrate: a) $\left.0.48 \cdot \Phi_{\mathrm{BEP}}, \mathrm{b}\right) 0.64 \cdot \Phi_{\mathrm{BEP}}, \mathrm{c}$ ) $0.8 \cdot \Phi_{\mathrm{BEP}}$ and d) $0.96 \cdot \Phi_{\mathrm{BEP}}$
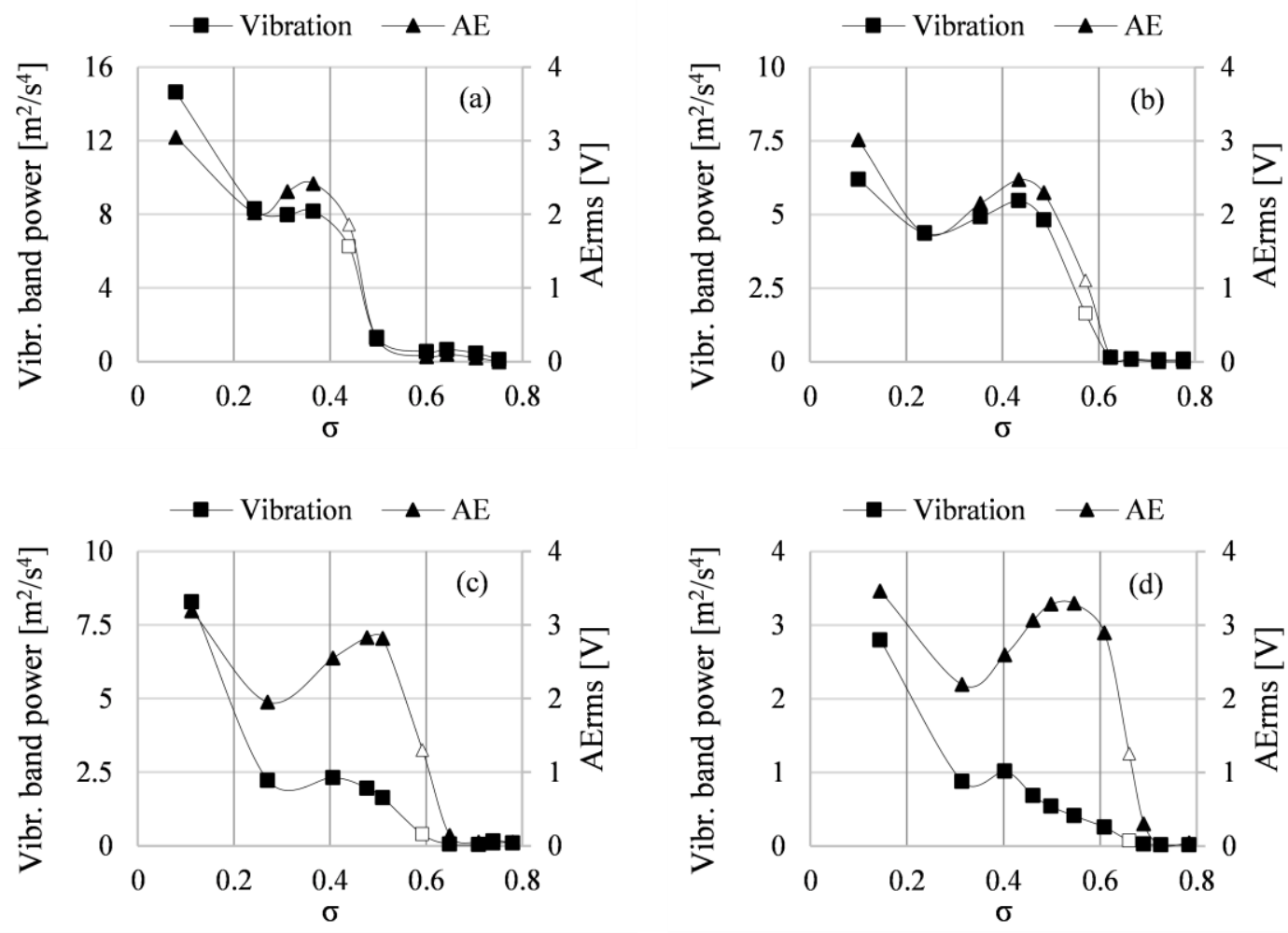

Figure 11 Noise and vibration as a function of $\sigma$ for Impeller 3 and flowrate: a) $\left.0.43 \cdot \Phi_{\mathrm{BEP}}, \mathrm{b}\right) 0.57 \cdot \Phi_{\mathrm{BEP}}, \mathrm{c}$ ) $0.71 \cdot \Phi_{\mathrm{BEP}}$ and d) $0.85 \cdot \Phi_{\mathrm{BEP}}$ 
From that point, as $\sigma$ takes even smaller values, the cavitation area enlarges and becomes more unstable and disintegrating at the edges, where bubbles are detached and collapsed in the flow stream. At these operating points that correspond to $\sigma_{\mathrm{m} 3}$ pictures in Figure 7, the $\mathrm{AE}_{\mathrm{RMS}}$ and the majority of the vibration band power measurements start to diminish (Figures 9 to 11). According to Gulich [25] the main cause of this decrease is the drop of the pressure differential between the pressure of the surrounding liquid and vapor pressure due to the increase of bubble volume and thus the rise of the compressibility of the medium. This noise and vibration behavior has also been observed and discussed by several authors $[6,9,10,11,12 \& 15]$. As $\sigma$ reduces towards the $\sigma_{\mathrm{FC}}$ value, the flow becomes extremely unsteady with complex cavitation pattern, as discussed in section 3.2. At this operating point impeller 1 behaves differently, in terms of noise and vibration, than the other two impellers, due to its better suction characteristics, discussed also in section 3.2 (cavitation region remains more concentrated and stable even at small $\sigma$ values). The vibration band power and $\mathrm{AE}_{\mathrm{RMS}}$ continue to decrease after the local maximum point, until the lowest measured $\sigma_{\mathrm{FC}}$ value (Figure 9). Hence, the compressibility mechanism discussed previously still prevails in this $\sigma$ range. On the contrary, the vibration band power and AE signals for impellers 2 and 3 increase inversely proportional to $\sigma$ value and maximize at the lowest tested value, $\sigma_{\mathrm{FC}}$ (Figs. 10 and 11). This behavior is possibly due to the result of two mechanisms that coexist at part load conditions. On the one hand, vibrations increase due to an increase in the blade incidence angle (Figure 4), and the intensity of the flow turbulence in the pump. According to [25-27 \& 29], in low specific speed machines operating at part load conditions the flow becomes three dimensional and strong recirculation regions are created in the impeller flow path. The large eddies formed at those recirculation areas excite pressure pulsations that raise the overall energy of the vibration signal, even for operating conditions with $\sigma$ value higher than $\sigma_{\mathrm{i}}$.

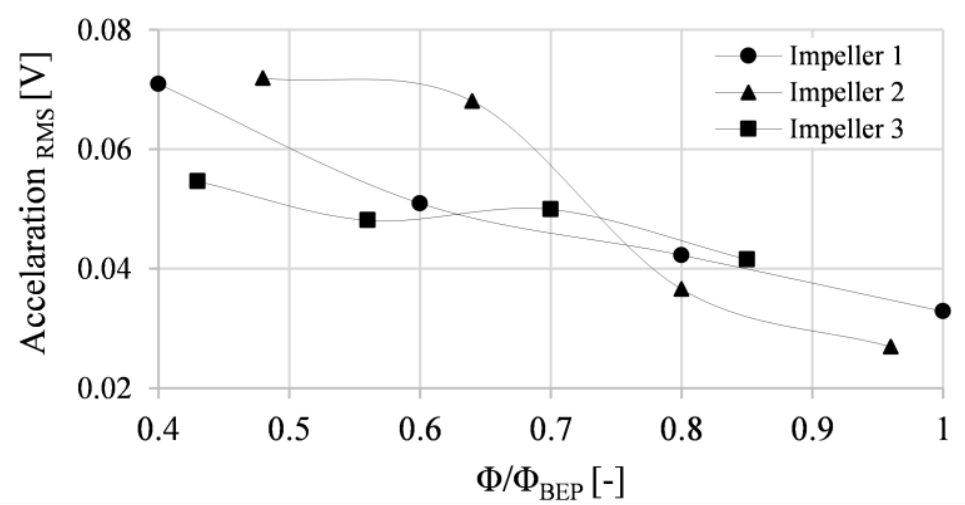

Figure 12 Vibration RMS in different flowrate fractions for $\sigma>0.75$ for the three impellers

In order to confirm this argument, the vibration RMS level for the flowrates tested here and at operating points without cavitation $(\sigma>0.75)$ was measured and the results are presented in Figure 12. It can be observed that the RMS level increases almost linearly as the flow rate reduces for all three impellers. These results agree with the findings of Alfayez [10] who identified the BEP of a large centrifugal pump using an AE sensor, and with Zhang [17] who measured the low frequency band vibration signals and both RMS values exhibited similar trend to the present study. Moreover, the above mechanism can also explain the higher vibration RMS values measured for impeller 3 compared to impeller 2 (Figs. 11 and 10, respectively), since the forward curved outlet shape of impeller 3 blades (Fig. 2) create more complex and non-uniform flow field in this impeller. The second instability mechanism at part load conditions, is due to the accelerated and highly cavitating flow that reenters the impeller inlet region after passing through the narrow clearance between the blades and the pump casing. According to Brennen [10] the backflow cavitation becomes more intense when the leakage flowrate becomes comparable with the pump flowrate, therefore at part load conditions. Finally, the $\mathrm{AE}_{\mathrm{RMS}}$ level variation with $\sigma$ exhibits a relatively similar pattern and values for the second 
and third impeller, and moreover, the corresponding curves remain comparable for all flowrates tested, as can be seen in Figs. 10 and 11. Therefore, the AE signals do not seem to be affected by the turbulence intensity and the backflow cavitation mechanisms. On the contrary, the results in Figs. 10 and 11 show a small decrease in $\mathrm{AE}_{\mathrm{RMS}}$ values as the flowrate is reduced. A possible explanation for this behavior is that the backflow cavitation disintegrates the main cavity formed at the blade suction side and distributes the bubbles within the entire flow passage (Fig. 7 , for $\sigma_{\mathrm{FC}}$ ), thus causing a smoothing effect on the $\mathrm{AE}$ signal. This effect becomes stronger as the backflow cavitation becomes more intense compared to the main flow, namely when the pump flowrate reduces. On the other hand, the cavitation region in impeller 1 remains more concentrated for the lowest $\sigma_{\mathrm{FC}}$ value tested (Fig. 7), and as a result, the $\mathrm{AE}_{\mathrm{RMS}}$ levels remain about the same for all flowrates (Fig. 9).

\section{Conclusions}

This study discusses the inception and development of cavitation in three different impellers of a centrifugal pump made from Plexiglas with the use of flow visualization, acoustic emissions and vibration monitoring. The total head, $\mathrm{AE}_{\mathrm{RMS}}$ and vibration band power curves are derived as function of the cavitation coefficient, $\sigma$, and interesting observations are made by comparing pictures taken from inside the impeller. More specifically, $\sigma_{i}$ values for cavitation inception increase with the flowrate, regardless of the impeller tested, due to the flow acceleration. The first bubbles always appear at the suction side of the leading edge of the blade, and the maximum values of noise and vibration are obtained much before the drop of pump head. Both the $\mathrm{AE}$ sensor and accelerometer were able to identify cavitation far before the total head drop operating point. The AE sensor was able to detect the appearance of the first visible bubble inside the impeller. The vibration trends are very similar to the $\mathrm{AE}$ trend, however, the lower frequency range used for the vibration analysis compared to $\mathrm{AE}$, resulted in delays in detection of the first bubble at some operating points.

Further decrease of $\sigma$ value results in an increasing number of bubbles and consequently intensifies the noise and vibration of the machine. However, after some point a decrease of $\sigma$ is found to temporarily reduce the noise and vibration emitted, because the vapor phase absorbs part of the sound. At the fully developed cavitation point the total pump head drops and the flow becomes very turbulent with large separation and cavitating regions. Comparisons between the three impeller results produce interesting conclusions on the effect of geometrical characteristics and of loading conditions in their cavitation behavior. At first, the lower incidence angle and the use of splitter blades of the first impeller results in milder noise and vibration characteristics through the entire $\sigma$ range tested. The increasing turbulence intensity and the backflow cavitation mechanism seem to be responsible for the observed increase in vibration band power at part load conditions, when the flow between the blades becomes less uniform, with strong 3D characteristics and internal recirculation zones. On the other hand, the $\mathrm{AE}_{\mathrm{RMS}}$ levels do not seem to be affected much by the flow turbulence, whereas the backflow cavitation mechanism might smoothen out at some degree at corresponding signals. The understanding of the internal flow characteristics could be significantly benefited from the use of a computational (CFD) model. Thus, the next step of the present work is focused on the development and validation of a CFD tool that could simulate pump operation under cavitating conditions.

\section{References}

[1] S. C. Li, Cavitation of hydraulic machinery (Vol. 1), World Scientific, 2000.

[2] Y. Tomita, A. Shima, High-speed photographic observations of laser-induced cavitation bubbles in water, Acustica, Vol. 71(3) (1985) 161-171.

[3] C. E. Brennen, Fundamentals of multiphase flow Cambridge University Press, 2005.

[4] G. M. Wood, J.S. Murphy, J. Farquhar, An Experimental Study of Cavitation in a Mixed Flow Pump Impeller, J. of Basic Engineering, Vol. 82(4) (1960) 929-940. 
[5] D. Papantonis, Hydrodynamic machines, hydrodynamic transmissions, Symeon, Athens, Greece (in Greek), 2009.

[6] P. J. McNulty, I.S. Pearsall, Cavitation Inception in Pumps, J. of Fluids Engineering, Vol. 104(1) (1982) 99-104.

[7] D. Ross, Mechanics of underwater noise, Elsevier, 2013.

[8] S. Gopalakrishman, Modern cavitation criteria for centrifugal pumps, Proc. of the Second Int. Pump Symp., Turbomachinery Laboratory, Texas A\&M University, College station, (1985).

[9] G. D. Neill, R. L. Reuben,P. M. Sandford, E.R. Brown, J. A. Steel, Detection of incipient cavitation in pumps using acoustic emission, Proceedings of the Institution of Mechanical Engineers, Part E: J. of process mechanical engineering, Vol. 211(4) (1997) 267-277.

[10] L. Alfayez, D. Mba, G. Dyson, Detection of incipient cavitation and the best efficiency point of a 2.2 MW centrifugal pump using Acoustic Emission, J. of Acoustic Emission, Vol. 22(2004) 77-82.

[11] C. E. Brennen, Hydrodynamic of pumps, Cambridge University Press, 2011.

[12] M. Chudina, Noise as an indicator of cavitation in a centrifugal pump, Acoustical Physics, Vol. 49(4) (2003) 463-474.

[13] J. Černetič, M. Čudina, Cavitation noise phenomena in centrifugal pumps, $5^{\text {th }}$ Congress of Alps-Adria Acoustics Assosiation, Petrcane, Croatia, 12-14 September 2012.

[14] M. Chudina, J. Prezelj, Detection of cavitation in operation of kinetic pumps. Use of discrete frequency tone in audible spectra, Applied Acoustics, 70(4) (2009) 540-546.

[15] J. Cernetič, J. Prezelj, M. Chudina, Use of noise and vibration signal for detection and monitoring of cavitation in kinetic pumps, The Journal of the Acoustical Society of America, Vol. 123(5) (2008) 3316-3316.

[16] M. Chudina, J. Prezelj, Detection of cavitation in situ operation of kinetic pumps: Effect of cavitation on the characteristic discrete frequency component, Applied acoustics, 70(9) (2009) 1175-1182.

[17] N. Zhang, M. Yang, B. Gao, Z. Li, Vibration characteristics induced by cavitation in a centrifugal pump with slope volute, Shock and Vibration, Vol. 2015(2015).

[18] O. Coutier-Delgosha, R. Fortes-Patella, L. Reboud, M. Hofmann, B. Stoffel, Experimental and numerical studies in a centrifugal pump with two-dimensional curved blades in cavitating condition, Journal of Fluids Engineering, 125.6 (2004) 970-978.

[19] H. Liu, D. Liu, Y. Wang, X. Wu, J. Wang, H. Du, Experimental investigation and numerical analysis of unsteady attached sheet cavitating flows in a centrifugal pump, Journal of Hydrodynamics, Ser. B 25.3 (2013) 370-378

[20] D. Zhang, W. Shi, D. Pan, M. Dubuisson, Numerical and Experimental Investigation of Tip Leakage Vortex Cavitation Patterns and Mechanisms in an Axial Flow Pump, Journal of Fluids Engineering. Vol. 137, 121103 (2015)

[21] G. Aggidis, http://www.lancaster.ac.uk/lureg.

[22] IEC 60193, Hydraulic turbines, storage pumps and pump turbines Model acceptance tests, (1999-2011).

[23] I. Farrance, R. Frenkel, Uncertainty of measurement: a review of the rules for calculating uncertainty components through functional relationships, The Clinical Biochemist Reviews, 33(2), 49 (2012).

[24] ISO (2009), 10816-7, Mechanical vibration - Evaluation of machine vibration by measurements on non-rotating parts - Part 7: Rotodynamic pumps for industrial applications, including measurements on rotating shafts.

[25] J. F. Gülich, Centrifugal pumps, Springer, 2008.

[26] V.S. Lobanoff, R. R. Ross, Centrifugal pumps: design and application, Elsevier, 2013.

[27] P. Welch, The use of fast Fourier transform for the estimation of power spectra: a method based on time averaging over short, modified periodograms, IEEE Transactions on audio and electroacoustics, 15(2) (1967) 70-73.

[28] L. Nelik, Centrifugal \& Rotary Pumps: Fundamentals with Applications, CRC Press, 1999.

[29] P. Hergt, J. Starke, Flow patterns causing instabilities in the performance curves of centrifugal pumps with vaned diffusers, In Proc. of the $2^{\text {nd }}$ Int. Pump Symp., (1985) 67-75. 